\title{
Assessment of visual quality and spatial accuracy of fast anisotropic diffusion and scan conversion algorithms for real-time three- dimensional spherical ultrasound
}

\author{
Qi Duan, Elsa D. Angelini, Andrew Laine \\ Department of Biomedical Engineering, Columbia University \\ New York, NY, USA.
}

\begin{abstract}
Three-dimensional ultrasound machines based on matrix phased-array transducers are gaining predominance for realtime dynamic screening in cardiac and obstetric practice. These transducer arrays acquire three-dimensional data in spherical coordinates along lines tiled in azimuth and elevation angles at incremental depth. This study aims at evaluating fast filtering and scan conversion algorithms applied in the spherical domain prior to visualization into Cartesian coordinates for visual quality and spatial measurement accuracy.

Fast 3D scan conversion algorithms were implemented with several orders of interpolation kernels. Downsizing and smoothing of sampling artifacts were integrated in the scan conversion process. In addition, a denoising scheme for spherical coordinate data with 3D anisotropic diffusion was implemented and applied prior to scan conversion to improve image quality. Reconstruction results under different parameter settings, such as interpolation kernel, scaling factor, smoothing options, and denoising, are reported. Image quality was evaluated on several data sets via visual inspections and measurements of the dimension of a cylindrical phantom object. Error measurements of the cylinder's radius, reported in this paper, showed that the proposed fast scan conversion algorithms can correctly reconstruct three-dimensional ultrasound in Cartesian coordinates under tuned parameter settings. Denoising via threedimensional anisotropic diffusion was able to greatly improve the quality of the resampled data without affecting the accuracy of spatial information after the modification of the introduction of a variable gradient threshold parameter.
\end{abstract}

Keywords: Real-time three-dimensional ultrasound, scan conversion, anisotropic diffusion, fast interpolation, spherical coordinates, matrix phased array, denoising.

\section{INTRODUCTION}

The development of three dimensional ultrasound techniques originated in the early 1980s. In the 1990s, Von Ramm and Smith developed a new real-time three-dimensional (RT3D) ultrasound probe based on a matrix phased array transducer ${ }^{1-2-3-4}$. This probe could scan a whole volume of interest in a fixed position at a rate of about $70 \mathrm{~ms}$ per volume, offering a faster and more reliable data acquisition protocol than free-hand and rotational ultrasound systems. Experiments reported in this study were performed with a Volumetrics $\odot$ transducer which records the data in spherical format. While this ultrasound machine is no longer commercially available the SONOS7500 3D ultrasound machine, based on a matrix-phased technology, has been recently introduced by Philips Medical Systems $\odot$. The availability of this type of three-dimensional technology from an international medical system manufacturer, may facilitate this technology to become the modality of choice for dynamic sonographic screening in cardiac and obstetric practice in the next few years.

Phased array transducers acquire echo lines in spherical coordinates in real-time when scanning a volume of interest. Manipulation of the data for conversion to Cartesian representation is required for visualization, border tracing and volume measurements. This operation is called scan conversion in this paper. In our previous work ${ }^{5}$, we developed a fast scan conversion algorithm, which combined coordinate transform, downsampling and smoothing to correct for acquisition artifacts and generate satisfactory image quality. In this paper, we present an experimental quantitative study testing our fast resampling tool with several interpolation kernels, smoothing options, resolution settings and addition of a pre-processing in spherical domain with anisotropic diffusion filtering. We evaluated image quality for 
different parameter settings both qualitatively and quantitatively via measurement of object dimensions on static phantom data.

\section{METHODOLOGY}

\subsection{Scan conversion algorithm}

Real-time three-dimensional (RT3D) is acquired by steering radio-frequency pulses in two orthogonal directions controlling the azimuth and elevation angles as illustrated in Figure 1. The data is stored in this geometry with the Volumetric (C) transducer and accessible to the user. The two steering angles span a $63^{\circ}$ angle with 64 positions symmetric around the axis of the transducer at $1^{\circ}$ increment. The radial positions starts at some preset depth (referred to as the skin depth) and acquires curved 'slices' every $0.308 \mathrm{~mm}$ with up to 512 slices stored by the machine, for each volume acquisition. Images corresponding to a constant depth along the transducer axis are called C-scan views. Fanshaped views orthogonal to the C-scan views are referred to as B-scan views.

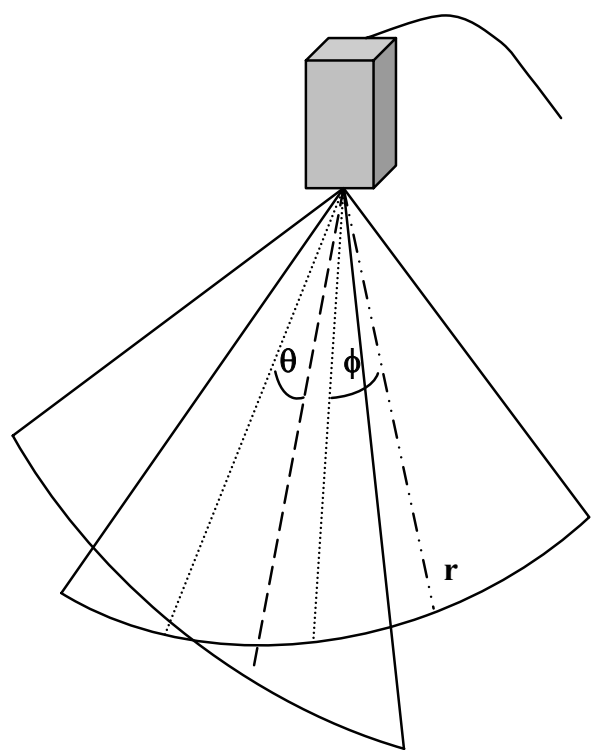

Figure 1: Geometry of acquisition of RT3D ultrasound data.

Lines are acquired in spherical coordinates $(r, \theta, \phi)$.

Scan conversion refers to the resampling of the RT3D data sets from spherical to Cartesian coordinates. The backward resampling process selected in this work is comprised of three steps: (1) coordinate transformation from the Cartesian grid to the spherical grid, (2) data interpolation on continuous spherical coordinate values, (3) data resampling back to the Cartesian grid. This process is illustrated in Figure 2 in two dimensions. The coordinate transformation was performed with standard formula to convert Cartesian to spherical coordinates. Data interpolation on the spherical data extrapolates the ultrasound values at continuous point locations via convolution with and an interpolation kernel of finite support. Theoretically, a band-limited signal sampled at a rate higher than its Nyquist frequency can be reconstructed perfectly via convolution with a sinc function. However, in discrete signal processing, only approximations of this function can be implemented for interpolation. 


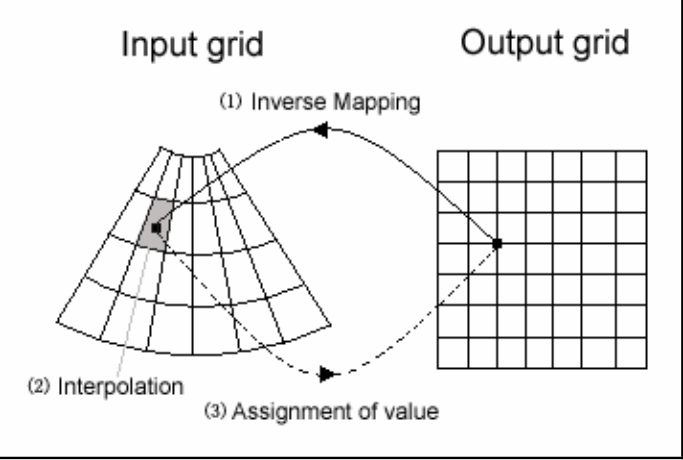

Figure 2: Illustration of scan conversion on 2D data. Resampling with backward mapping is performed from radial grid coordinates $(r, \theta)$ to Cartesian grid coordinates $(x, y)$.

We tested in a previous study ${ }^{5}$ interpolation kernels of different order (related to smoothness) and support size, including a nearest neighbor (NN) filter, a linear interpolation (LI) filter, a cubic convolution (CC) filter, a Hann windowed sinc function (Hann), a Hamming windowed sinc function (Hamming), a Lanczos window (Lan), and a Gaussian window (Gauss). The one-dimensional shape of these filters is illustrated in Figure 3 along with the theoretically optimal sinc function.
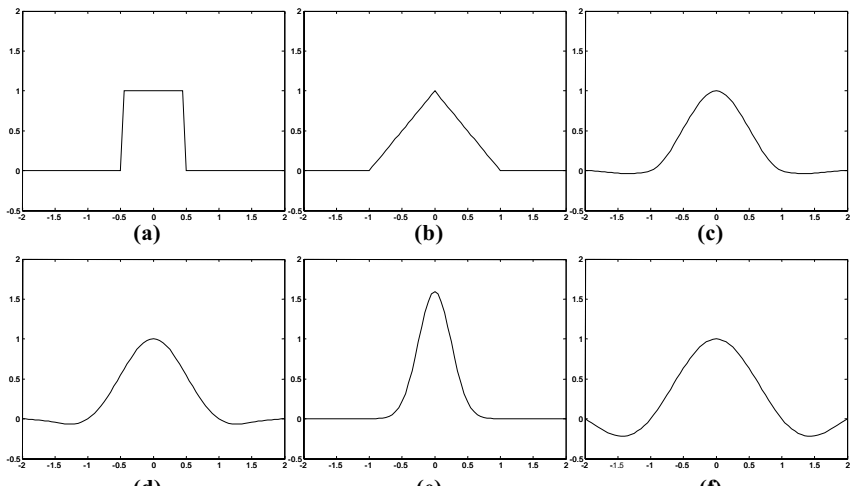

(d)

(f)

Figure 3: 1D profiles of interpolation kernels on interval [-2 2] for: (a) nearest neighbor, (b) linear, (c) cubic $(a=-0.5)$, (d) Hamming windowed sinc, (e) Gaussian $(\sigma=0.5)$, (f) theoretically optimal sinc kernel.

The filter width (i.e. support size) and the shape parameters for each interpolation kernel were optimized in a previous study ${ }^{5}$ whose results are summarized in Table 1.

\begin{tabular}{|c|c|c|c|c|c|c|c|}
\hline & NN & LI & CC & Hann & Hamming & Lan & Gauss \\
\hline Filter width & 1 & 1 & 2 & 3 & 3 & 3 & 3 \\
\hline $\begin{array}{c}\text { Shape } \\
\text { Parameter }\end{array}$ & - & - & -0.5 & - & - & - & $\sigma=1$ \\
\hline
\end{tabular}

Table 1: Optimal parameter settings for the interpolation kernels.

Smoothing can be introduced in the interpolation process by weighting the kernel coefficients. Downscaling of the data, referring to the axial resolution of acquisition equal to $0.308 \mathrm{~mm}$ can also be used to control the reconstructed pixel size and reduce at the same time acquisition artifacts while avoiding aliasing. We consider in our discussion scale $=1$ for a resampling of the data that preserves the highest spatial resolution acquired corresponding to a voxel size of $(0.308 \mathrm{~mm})^{3}$. 


\subsection{Anisotropic Filtering}

The presence of speckle noise patterns makes the interpretation of ultrasound images, either by a human operator or with a computer-based system, very difficult. It is highly desirable for certain applications such as automatic segmentation, to apply some denoising prior to scan conversion in order to remove speckle noise artifacts and improve signal homogeneity within distinct anatomical tissues. A number of methods have been proposed to de-noise and improve the ultrasound image quality including temporal averaging, median filtering, maximum amplitude writing (temporal dilation), adaptive speckle reduction (ASR) (statistical enhancement) ${ }^{6-7-8-9-10}$, adaptive weighted median filter $(\mathrm{AWMF})^{11}$, homomorphic Wiener filtering, and wavelet shrinkage (WS) ${ }^{12-13}$. Most of these methods suffer from either insufficient denoising, image quality degradation or large computational cost. Furthermore, some of them require raw "rf" data, prior to logarithmic compression" ${ }^{14}$. Our group has presented previous work on applying brushlet denoising in spherical coordinates to RT3D cardiac ultrasound prior to resampling of the data ${ }^{15}$. Experiments on phantom and clinical cardiac data sets have shown excellent performance of the method. However, the main limitation of this type of denoising remains the computational cost that prevents for the moment its implementation for real-time visualization applications in clinical practice.

In this context we have investigated the performance of a more computationally efficient denoising filter based on anisotropic diffusion for data represented in spherical coordinates. A similar framework can be found in the work of Abd-Elmoniem et al. ${ }^{14-16}$ who used two-dimensional anisotropic filtering in radial coordinates. Anisotropic diffusion methods are very efficient for speckle reduction in ultrasound and radar images. In recent publications, Yu and Acton $^{17-18}$ applied their speckle reducing filter on synthetic aperture radar images and compared their performance to Lee and Kuan filters and Frost filters. These filters are all derived from anisotropic diffusion. Finally we cite Montagnat et al who applied a three-dimensional anisotropic diffusion filter for rotational cardiac 3D ultrasound $\operatorname{data}^{19}$.

Anisotropic diffusion methods apply the following heat-diffusion type of dynamic equation to the gray levels of a given 3D image data $I(x, y, z, t)$ :

$$
\frac{\partial I}{\partial t}=\operatorname{div}(c(x, y, z, t) \nabla I)
$$

where $c(x, y, z, t)$ is the diffusion parameter, div denotes the divergence operator, and $\nabla I$ denotes the gradient of the image intensity.

In the original work of Perona and Malik ${ }^{20-21}$, the concept of anisotropic diffusion was introduced with the selection of a variable diffusion parameter, function of the gradient of the data:

$$
c(x, y, z, t)=g(|\nabla I(x, y, z, t)|)
$$

We used the diffusion function proposed by Weickert ${ }^{22}$ defined as:

$$
g(x, \lambda)=\left\{\begin{array}{ll}
1 & x \leq 0 \\
1-e^{-\frac{3.315}{(x / \lambda)^{4}}} & x>0
\end{array} .\right.
$$

The parameter $\lambda$ serves as a gradient threshold, defining edge points $x_{k}$ as locations where $\left|\nabla I_{x_{k}}\right|>\lambda$. This bellshaped diffusion function acts as an edge-enhancing filter, with high diffusion values in smooth areas and low values at edge points. The structure of the diffusion tensor with separate weights for each dimension enables to control the direction of the diffusion process, with flows parallel to edge contours.

Initial experiments on RT3D spherical data showed that selecting a low gradient threshold enabled the filtering to remove speckle noise components but did not remove blocky sampling artifacts as seen in Figure 5. On the other hand, a high gradient threshold value was able to smooth out the sampling artifacts but was not efficient at removing noisy components. 
As the diffusion process evolves, image data properties change and it is desirable to modify the gradient threshold parameter value. In their paper, Montagnat et al. report a decrease in the value of significant edges as the homogeneous regions in the ultrasound data are filtered. They therefore chose to decrease the threshold gradient in time and proposed values based on a fraction of the cumulative histograms of the data gradients recomputed at each iteration of the diffusion process.

In our case, with more granular images, as the diffusion evolves in time we obtain higher values of gradient at significant edges. Indeed as the data is diffused, gradients corresponding to real objects produce much higher values than gradients due to the smoothed speckle noise oscillations. After trying different strategies for setting the gradient threshold value, we used a linear model where:

$$
\lambda(t)=\lambda_{0}+a t
$$

with $\lambda_{0}$ an initial gradient value, $a$ is a slope parameter and $t$ is the time iteration index. Parameters were set empirically for the data sets processed.

\section{DATA}

In order to evaluate our scan conversion and denoising methods we performed a qualitative visual inspection as well as quantitative measurements on both phantom and clinical data sets.

\subsection{Phantom objects}

The phantom consisted of two cylindrical objects with a change of radius in the axial direction as illustrated in Figure 4. The larger radius was equal to $10 \mathrm{~mm}$. The medium in the phantom box varied for each cylindrical object, mimicking tissues with various contrast measured in terms of signal to noise ratio (SNR).

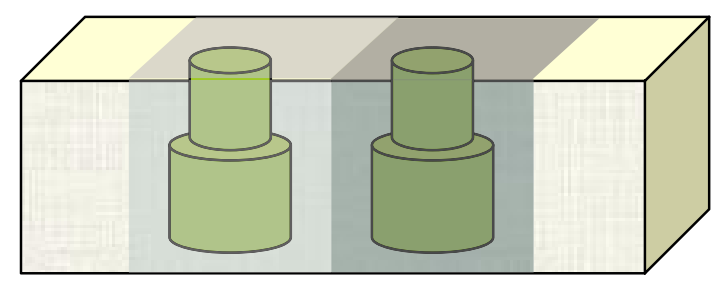

Figure 4: Phantom objects with two cylindrical objects embedded in medium with different SNR.

We focused our experiments on a $\mathrm{SNR}=2 \mathrm{db}$, corresponding to good contrast. The clinical application of our study focused on echocardiographic data with excellent contrast between the myocardium muscle and the ventricular blood pool. The phantom was screened with a RT3D Volumetrics $($ machine with acquisition parameters identical to clinical settings.

\subsection{In-vitro cardiac tissue}

A second experiment was performed using an in-vitro phantom consisting of a cardiac muscle sample placed in a tank of water. The muscle sample was cut as a cube of cross section equal to $10 \mathrm{~mm}^{2}$. The cardiac sample was screened with a RT3D Volumetrics $\subset$ machine with acquisition parameters identical to clinical settings.

\subsection{Clinical data}

We also applied our filtering methods to echocardiographic data set of a healthy volunteer on a single time frame. This data set was also denoised with a brushlet expansion as detailed in $^{23}$ from our previous study. 


\section{RESULTS}

We performed several experiments with different parameter settings for the scan conversion, with and without smoothing. Pre-processing of the data with anisotropic diffusion was tested for each data set with the following parameters:

- $\lambda_{0}=5, \mathrm{a}=10, \Delta \mathrm{t}=0.05,10$ iterations for the phantom,

- $\lambda_{0}=6, \mathrm{a}=10, \Delta \mathrm{t}=0.05,10$ iterations for the muscle sample,

- $\lambda_{0}=6, \mathrm{a}=10, \Delta \mathrm{t}=0.05,10$ iterations for the clinical data set.

The threshold value $\lambda_{0}$ was set equal to the average gradient of the background speckle noise, estimated on a manually-selected region of interest. A second region of interest inside the object to smooth was selected to check the maximum gradient value. This value was used to set the slope parameter so that after $N$ iterations, the threshold value reaches the maximum gradient value inside the object. In our experiments, we ran 10 iterations with a small time step and set $a$ to 10 so that the final gradient threshold value $\lambda$ was around 100 , for the maximum gradient value inside the phantom objects and the myocardium.

Computation times of the different experiments are reported in Table 2, for an implementation in $\mathrm{C}$, running on a PC dual Pentium IV $2.2 \mathrm{GHz}$ processors workstation with $1.5 \mathrm{GBytes}$ of RAM.

\begin{tabular}{|c|c|c|c|c|c|c|}
\hline Data & $\begin{array}{c}\text { Matrix size } \\
\text { in spherical } \\
\text { coordinates }\end{array}$ & $\begin{array}{c}\text { Scale of } \\
\text { scan } \\
\text { conversion }\end{array}$ & $\begin{array}{c}\text { Smoothing } \\
\text { option for } \\
\text { scan } \\
\text { conversion }\end{array}$ & $\begin{array}{c}\text { Matrix size in } \\
\text { Cartesian } \\
\text { coordinates }\end{array}$ & $\begin{array}{c}\text { Diffusion } \\
\text { computation } \\
\text { time for one } \\
\text { iteration } \\
\text { (seconds) }\end{array}$ & $\begin{array}{c}\text { Scan } \\
\text { conversion } \\
\text { computation } \\
\text { time } \\
\text { (seconds) }\end{array}$ \\
\hline $\begin{array}{c}\text { Phantom } \\
\text { object }\end{array}$ & $64 \times 64 \times 373$ & 1 & No & $389 \times 389 \times 376$ & 0.38 & 106 \\
\hline Cardiac tissue & $64 \times 64 \times 258$ & 1 & No & $274 \times 274 \times 261$ & 0.25 & 37 \\
\hline Clinical exam & $64 \times 64 \times 438$ & 1 & No & $454 \times 454 \times 441$ & - & 169 \\
\hline Clinical exam & $64 \times 64 \times 438$ & 2 & No & $228 \times 228 \times 221$ & - & 21 \\
\hline Clinical exam & $64 \times 64 \times 438$ & 2 & Yes & $228 \times 228 \times 221$ & - & 23 \\
\hline Clinical exam & $64 \times 64 \times 438$ & 1 & No & $454 \times 454 \times 441$ & 0.51 & 169 \\
\hline
\end{tabular}

Table 2: Computation times for the different experiments for the scan conversion and the anisotropic diffusion algorithms.

Overall, anisotropic diffusion filtering with 10 iterations required up to 5 seconds of computation time. Scan conversion required about 2 minutes at maximum resolution and 30 seconds at half resolution. These results show very fast computation speeds of the filtering process applicable for routine use.

\subsection{Image quality from scan conversion}

Qualitative assessment of phantom and clinical images reconstructed with the fast interpolation kernels showed minor image quality degradation between scan conversion with full resolution and downscaling with a factor of tow. The smoothing parameters had minor effects for all interpolation kernels when downscaled by a factor of 2 . These results are illustrated in Figure 5. 


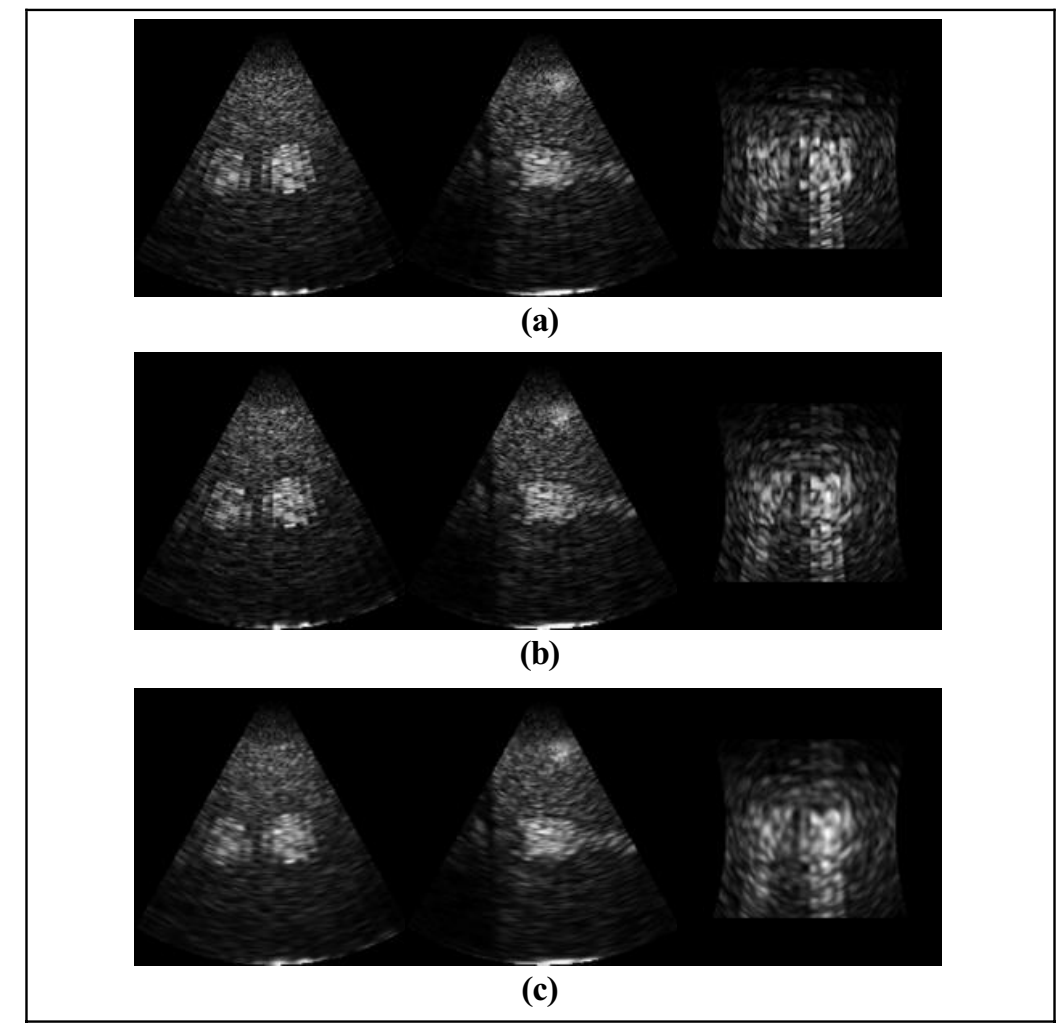

Figure 5: Results of scan conversion on three orthogonal views of a cylindrical phantom object with different smoothing and scaling parameters: (a) Scale $=1$ and no smoothing, (b) Scale $=2$ and no smoothing. (c) Scale $=2$ with smoothing.

Higher downscaling factors introduced noticeable degradation of spatial resolution (corresponding to a voxel sizes above $1 \mathrm{~mm}$ ) and are not recommended for clinical applications.

\subsection{Image quality from anisotropic diffusion}

Qualitative comparison of phantom data reconstructed with and without anisotropic diffusion showed very good performance of the non-linear filter at effectively removing speckle noise granularity in low intensity background areas using an initial low gradient threshold and effectively correcting for the high-intensity object inhomogeneity using a higher gradient threshold as the diffusion process evolved. As illustrated in Figure 6, controlling the diffusion process with a time-varying threshold gradient greatly improved filtering performance when compared to a fixed threshold setting. This result was particularly significant in view of the challenge that speckle denoising represents and the simplicity of the design of the proposed algorithm. Speckle noise components are typically correlated with the underlying signal leading to spatially varying statistics and blocky artifacts showing strong edges. These properties make removal of speckle components a particularly challenging task where traditional filtering typically fail. A recent work from $\mathrm{Yu}$ and Acton $^{18}$ designed a speckle reducing anisotropic diffusion (SRAD) filter that proposed a diffusion coefficient based on local statistics of the ultrasound signal. The proposed filter has a more refined design but was only proposed in 2D and with results only illustrated in one carotid artery ultrasound image. Nevertheless the authors draw similar conclusion regarding the potential of anisotropic diffusion in reducing speckle noise components with a proper design of the diffusion coefficient. 


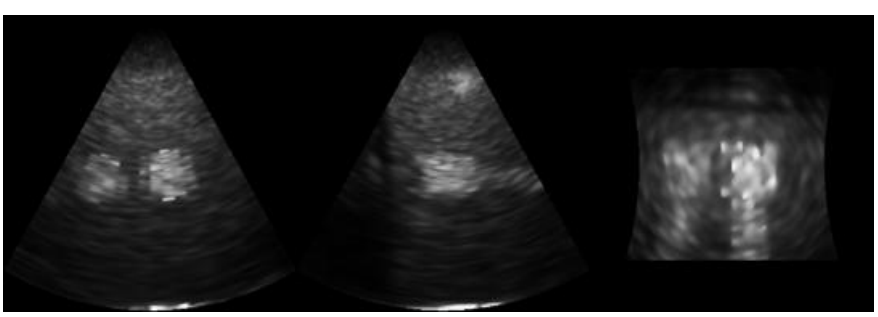

(a)

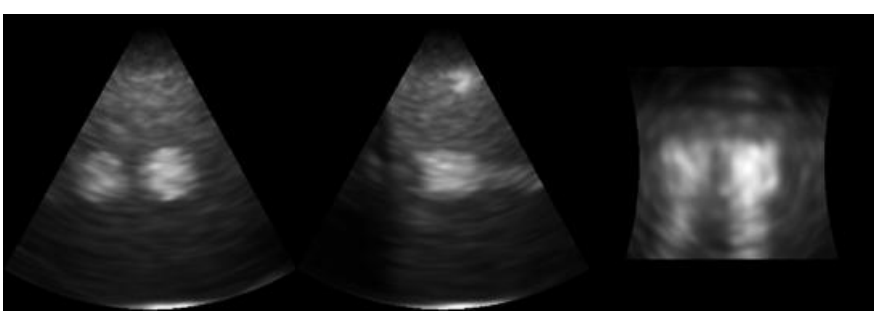

(b)

Figure 6: Anisotropic diffusion filtering and scan conversion with a cubic interpolation kernel without smoothing or downscaling. Results are presented for three orthogonal views of a cylindrical phantom object. (a) Anisotropic diffusion with a fixed gradient threshold. (b) Anisotropic diffusion with a variable gradient threshold.

We observed similar image quality improvement using the time-varying anisotropic diffusion filter on the static myocardium tissue sample data set and the clinical data of the left ventricle of a healthy volunteer. These results are illustrated in Figure 7 and Figure 8 and confirmed the very good performance of the filter at improving image quality in the spherical domain prior to scan conversion.

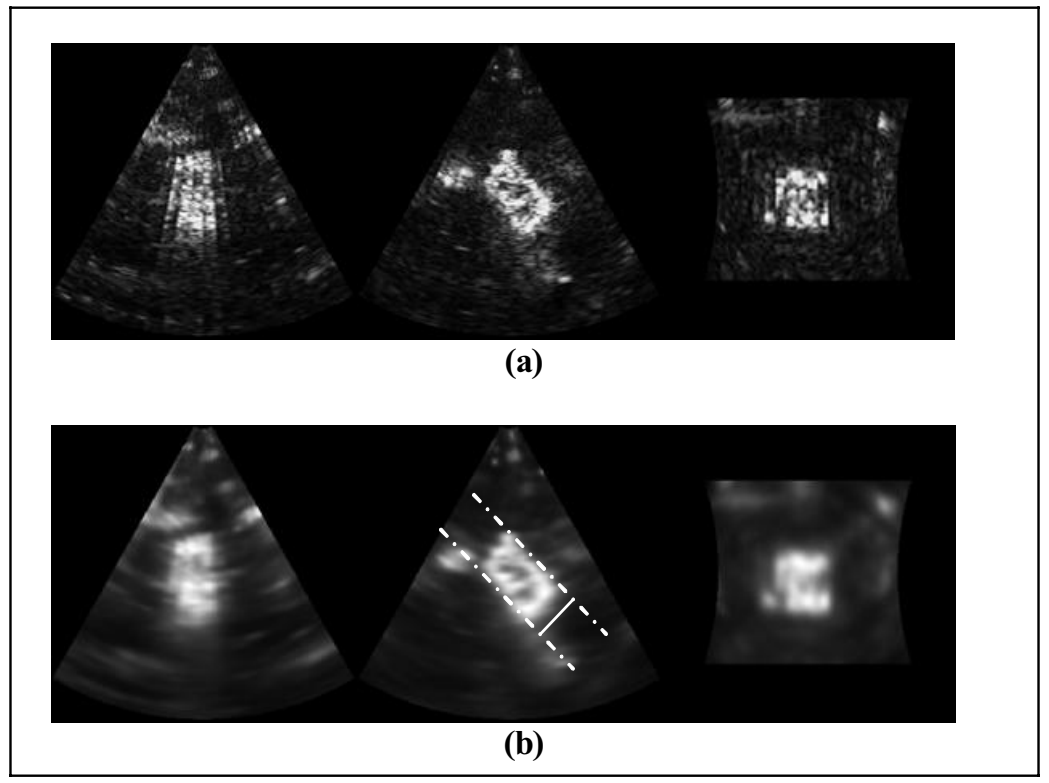

Figure 7: Anisotropic diffusion filtering and scan conversion with a cubic interpolation kernel without smoothing or downscaling. Results are presented for three orthogonal views of an in-vitro myocardium tissue sample. (a) Original data. (b) Data after anisotropic diffusion with a variable gradient threshold. Note in the Bscan view the indication of the average dimension measurement performed on the filtered data. 
Comparing anisotropic diffusion to three-dimensional brushlet denoising, we observed good performance of the anisotropic diffusion filter at enhancing the ventricular cavity and smoothing the myocardium tissue. Such improvement in image quality can greatly improve analysis of the data for volume rendering or segmentation for example. Brushlet denoising on the other hand better preserved the texture of the myocardium muscle and some details in the anatomical contours of the ventricle. While both denoising methods were able to eliminate the granular noise texture generated during the data acquisition by speckle interferences, brushlet denoised data showed less attenuation of the blocky acquisition artifacts observed in B-scan views when compared to anisotropic diffusion.

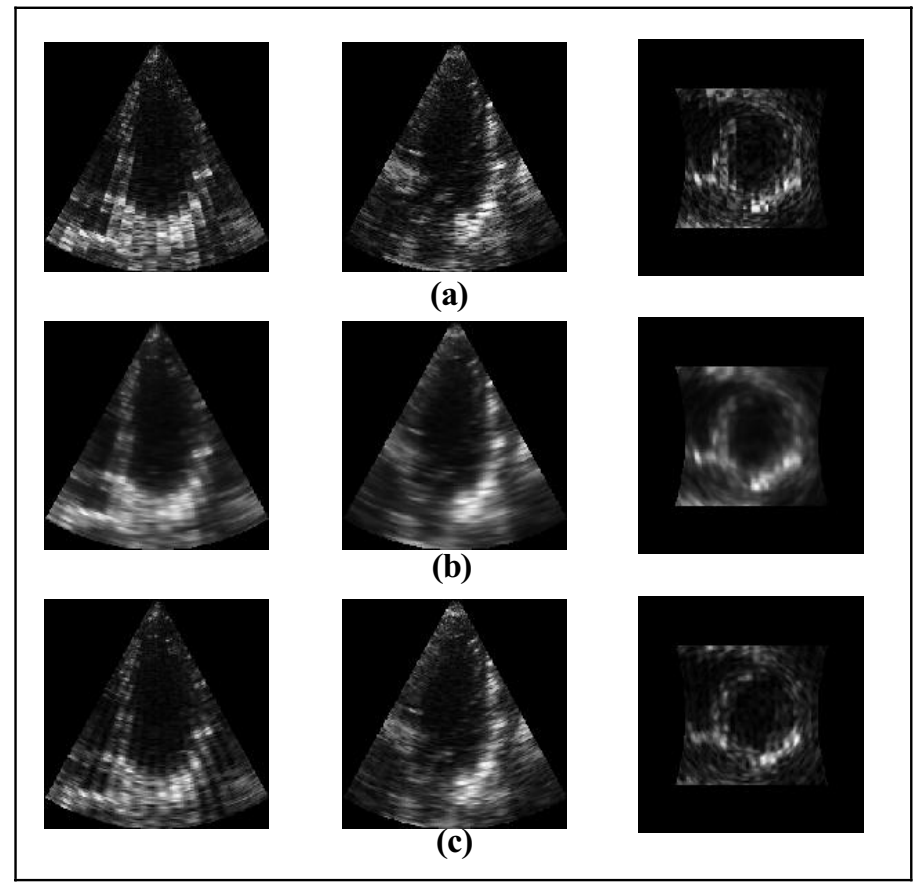

Figure 8: Anisotropic diffusion filtering and scan conversion with a cubic interpolation kernel without smoothing or downscaling. Results are presented for three orthogonal views of an in-vivo data set of a left ventricle. (a) Original data. (b) Anisotropic diffusion with a variable gradient threshold. (c) Denoising with brushlet thresholding in space.

Qualitative evaluation of the anisotropic diffusion filters lead to the conclusion that it offered denoising performance that compared well to more elaborated methods such as brushlet spatial denoising with a tremendous gain in computation load. Moreover, image quality from scan-conversion of volumes after anisotropic diffusion was almost insensitive to the choice of the interpolation kernel. These results suggest that 3D non-linear anisotropic diffusion can be combined with fast interpolation methods using lower order kernels to optimize the display process and significantly improve image quality at low computational cost.

\subsection{Measurements of object dimensions}

A quantitative study was performed on the cylindrical phantom objects to evaluate the accuracy of measurement obtained with the different parameter settings and filters reported above. Radius of the biggest cylinder in the phantom dataset was estimated via manual tracing on $10 \mathrm{~B}$-scan cross-sections by a single operator. Average radius measurements $(R)$ along with relative errors $(E r r)$ are reported in Table 3. 


\begin{tabular}{|c|c|c|c|c|c|c|c|c|c|c|}
\hline & \multicolumn{2}{|c|}{ Data 1 } & \multicolumn{2}{c|}{ Data 2 } & \multicolumn{2}{c|}{ Data 3 } & \multicolumn{2}{c|}{ Data 4 } & \multicolumn{2}{c|}{ Data 5 } \\
\cline { 2 - 12 } & $\mathbf{R}(\mathbf{m m})$ & Err \% & $\mathbf{R}(\mathbf{m m})$ & Err \% & $\mathbf{R}(\mathbf{m m})$ & Err \% & $\mathbf{R}(\mathbf{m m})$ & Err \% & R (mm) & Err \% \\
\hline NN & 10.03 & 0.33 & 9.89 & -1.12 & 10.09 & 0.89 & 10.18 & 1.79 & 9.97 & -0.33 \\
\hline LI & 10.10 & 1.01 & 9.97 & -0.29 & 10.07 & 0.74 & 10.19 & 1.86 & 10.11 & 1.09 \\
\hline CC & 10.06 & 0.61 & 10.16 & 1.60 & 10.06 & 0.56 & 9.99 & -0.09 & 9.96 & -0.42 \\
\hline Hann & 10.10 & 1.03 & 10.12 & 1.23 & 9.97 & -0.27 & 9.96 & -0.45 & 10.04 & 0.35 \\
\hline Hamming & 10.09 & 0.87 & 9.84 & -1.57 & 10.10 & 1.02 & 9.91 & -0.09 & 10.10 & 0.97 \\
\hline Lan & 10.20 & 2.01 & 9.92 & -0.83 & 10.01 & 0.14 & 10.02 & 0.18 & 9.94 & -0.63 \\
\hline Gauss & 9.97 & -0.28 & 9.95 & -0.47 & 10.00 & -0.03 & 9.66 & -3.67 & 9.85 & -1.48 \\
\hline
\end{tabular}

Table 3: Measurements of a cylinder radius (exact value is $10 \mathrm{~mm}$ ) for: Data 1: Original data-scale=1-no smoothing; Data 2: Original data-scale=2-no smoothing; Data 3: Original data-scale=2-smoothing; Data 4: Diffused data (thresholds=5, 10 iterations)-scale=1-no smoothing; Data 5: Diffused data (variable threshold, 10 iterations)-scale $=1$-no smoothing.

Results showed that the combination of interpolation kernel, scaling factor, smoothing options and pre-processing with anisotropic filtering had some influence on the accuracy of the object size measurement. The combination of processing parameters leading to the smallest measurement error (equal to $0.03 \%$ ) was: downscaling by a factor of 2 , smoothing and a Gaussian interpolation kernel. With anisotropic diffusion, variable thresholding systematically reduced measurement errors with an optimal configuration at $0.3 \%$ error for scale 1 using a nearest neighbor interpolation.

We performed a similar object size measurement experiment on the in-vitro cardiac muscle phantom. The muscle sample was cut to have a cross section of $10 \mathrm{~mm}^{2}$. Unfortunately, the sample was not carefully positioned during the scanning so that $\mathrm{C}$-scan views contained a cross sectional view bigger than the orthogonal cross section of the sample. We performed oblique measurements on B-scan views as illustrated in Figure 7(c) and obtained an average measurement of $10.77 \mathrm{~cm}$, which seems to be a reasonable measure given the potential errors in cutting the myocardium sample and positioning of the sample within the scanned volume of interest.

These quantitative measurements can help in selecting the best parameter settings for filtering and scan conversion. They also showed that a measurement precision of $1 \mathrm{~mm}$ could be achieved on objects and in-vitro myocardium samples with three-dimensional ultrasound despite known limitations of hardware transducer design ultrasound physics and interferences.

\section{CONCLUSION}

A fast three-dimensional scan conversion algorithm combined with smoothing and anti-aliasing filtering for downsampling operations was introduced for spherical three-dimensional ultrasound data. In addition, a fast denoising method based on anisotropic diffusion was introduced with a novel time varying gradient-threshold scheme.

Experiments were performed with a Volumetrics $($ $)$ matrix-phased array transducer on static objects, in-vitro myocardium muscle sample and clinical data sets.

Filtering performance was assessed in terms of visual quality and for quantitative measurements of the phantom object dimensions. Our quantitative study showed that very high measurement accuracy could be achieved and required suitable parameter settings of the scan conversion method, while visual quality was similar for all interpolation kernels. The study also showed a great improvement in visual quality when applying the anisotropic diffusion prior to scan conversion and a comparable accuracy of measurements even with first order interpolation kernels. These results reveal the critical importance of the scan conversion algorithm design. They also demonstrate that with careful reconstruction of the data in Cartesian coordinates, three-dimensional matrix-phased array ultrasound modality can provide high spatial accuracy, around $1 \mathrm{~mm}$, in a real-time acquisition mode. Anisotropic diffusion can preserve this spatial accuracy while denoising the data for high-quality visual display and three dimensional rendering. 


\section{ACKNOWLEDGEMENTS}

We would like to thank Dr. Von Ramm from Duke University, Durham, NC for stimulating conversations on the subject. We would also like to Dr. Shunichi Homma, Dr. Kumiko Hirata and Dr. Ryo Otsuka from the Echocardiography Laboratories at Columbia Presbyterian Hospital for providing the ultrasound data sets.

\section{REFERENCES}

1. O. V. Ramm and S. W. Smith, "Real time volumetric ultrasound imaging system." Journal of Digital Imaging, Vol. 3, No. 4, pp. 261-266, 1990.

2. J. T. Yen and S. W. Smith, "Real-time rectilinear volumetric imaging." IEEE Transactions on Ultrasonics, Ferroelectrics, and Frequency Control, Vol. 49, No. No. 1, pp. 114-124, 2002.

3. S. W. Smith, H. G. Pavy and O. T. Ramm, "High-speed ultrasound volumetric imaging system. I: Transducer design and beam steering." IEEE Transactions on Ultrasound, Vol. 38, No. No. 2, pp. 100-108, 1991.

4. S. W. Smith, H. G. Pavy and O. T. Ramm, "High-speed ultrasound volumetric imaging system. II: Parallel processing and image display." IEEE Transactions on Ultrasound, Vol. 38, No. No. 2, pp. 109-115, 1991.

5. Q. Duan, E. D. Angelini, T. Song and A. Laine, "Fast interpolation algorithms for real-time three-dimensional cardiac ultrasound", IEEE EMBS Annual International Conference, pp. 1192-1195, Cancun, Mexico, 2003.

6. J. C. Bamber and C. Daft, "Adaptive filtering for reduction of speckle in ultrasonic pulse-echo images." Ultrasonics, Vol. 24, No. 1, pp. 41-44, 1986.

7. J. C. Bamber and G. Cook-Martin, "Texture analysis and speckle reduction in medical echography", SPIE, pp. 120-127, 1987.

8. J. C. Bamber and J. V. Philips, "Real-time implementation of coherent speckle suppression in B-scan images." Ultrasonics, Vol. 29, No. 3, pp. 218-224, 1991.

9. D. C. Crawford, D. S. Bell and J. C. Bamber, "Implementation of ultrasound speckle filters for clinical trial", Proceedings of IEEE Ultrasonic Symposium, pp. pp. 1589-1592, 1990.

10. D. C. Crawford, D. S. Bell and J. C. Bamber, "Compensation for the signal processing characteristics of ultrasound B-mode scanners in adaptive speckle reduction." Ultrasound in Medicine and Biology, Vol. 19, No. 6, pp. 469-485, 1993.

11. T. Loupas, W. N. Mcdicken and P. L. Allan, "An adaptive weighted median filtering for speckle suppression in medical ultrasonic images." IEEE Transactions on Circuits and Systems, Vol. 36, pp. 129-135, 1989.

12. X. Hao, S. Gao and X. Gao, "A novel multiscale nonlinear thresholding method for ultrasonic speckle suppressing." IEEE Transactions on Medical Imaging, Vol. 18, No. 9, pp. 787 - 794, 1999.

13. X. Zong, A. F. Laine and E. A. Geiser, "Speckle reduction and contrast enhancement of echocardiograms via multiscale nonlinear processing." IEEE Transactions on Medical Imaging, Vol. 17, No. 4, pp. 532-540, 1998.

14. K. Z. Abd-Elmoniem, A.-B. M. Youssef and Y. M. Kadah, "Real-time speckle reduction and coherence enhancement in ultrasound imaging via nonlinear anisotropic diffusion." IEEE Transactions on Biomedical Engineering, Vol. 49, No. 9, pp. 997-1014, 2002.

15. D. R. Gersony, E. D. Angelini, J. Donis, C. Dimayuga, R. J. Barst, R. Saouaf, M. D. Tullio, A. Laine and S. Homma, "Brushlet analysis technique improves real-time three dimensional calculation of right ventricular function in primary pulmonary hypertension patients", Proceedings of the Scientific Session of the American College of Cardiology, Atlanta, Georgia, USA, 2002.

16. K. Z. Abd-Elmoniem, Yasser M. Kadah and A.-B. M. Youssef, "Real time adaptive ultrasound speckle reduction and coherence", International Conference on Image Processing, pp. 172-175, Vancouver, BC, Canada, 2000.

17. Y. Yu and S. T. Acton, "Segmentation of ultrasound imagery using anisotropic diffusion", Asilomar Conference on Signals, Systems and Computers, pp. 1151-1155, 2001.

18. Y. Yu and S. T. Acton, "Speckle reducing anisotropic diffusion." IEEE Transactions on Image Processing, Vol. 11, No. 11, pp. 1260-1270, 2002.

19. J. Montagnat, M. Sermesant, H. Delingette and e. al, "Anisotropic filtering for model-based segmentation of 4D cylindrical echocardiographic images." Pattern Recognition Letters, Vol. 24, pp. 815-828, 2003.

20. P. Perona and J. Malik, "Scale space and edge detection using anisotropic diffusion", IEEE Workshop on Computer Vision, pp. 16-22, 1987. 
21. P. Perona and J. Malik, "Scale-space and edge detection using anisotropic diffusion." IEEE Transactions on Pattern Analysis and Machine Intelligence, Vol. 12, No. 7, pp. 629-639, 1990.

22. J. Weickert, B. M. t. H. Romeny and M. A. Viergever, "Efficient and reliable schemes for nonlinear diffusion filtering." IEEE Transactions on Image Processing, Vol. 7, No. 3, pp. 398-410, 1998.

23. E. Angelini, A. Laine, S. Takuma, J. Holmes and S. Homma, "LV volume quantification via spatio-temporal analysis of real-time 3D echocardiography." IEEE Transactions on Medical Imaging, Vol. 20, No. 6, pp. 457-469, 2001. 